Instructions for authors, subscriptions and further details:

\title{
http://mcs.hipatiapress.com
}

\section{Licencias de Paternidad y Permisos Parentales en América Latina y el Caribe. Herramientas Indispensables para Propiciar la Mayor Participación de los Padres en el Cuidado de los Hijos e Hijas}

Carina Lupica ${ }^{1}$

1) Observatorio de la Maternidad, Argentina

Date of publication: October $21^{\text {st }}, 2016$

Edition period: February 2017 - June 2017

To cite this article: Lupica, C. (2016). Licencias de paternidad y permisos parentales en América Latina y el Caribe. Herramientas indispensables para propiciar la mayor participación de los padres en el cuidado de los hijos e hijas. Masculinities and Social Change,5(3),295-320. doi:

10.17583/MCS.2016.2083

To link this article: http://doi.org/10.17583/MCS.2016.2083

PLEASE SCROLL DOWN FOR ARTICLE

The terms and conditions of use are related to the Open Journal System and to Creative Commons Attribution License (CC-BY). 
MCS - Masculinities and Social Change Vol. 5 No. 3 October 2016

pp. 295-320

\section{Paternity and Parental Leave in Latin America and the Caribbean. Essential Tools to Promote Greater Participation of Fathers in the Care of Children}

Carina Lupica

Observatorio de la Matenidad

\section{Abstract}

Institutional reform becomes necessary to accompany the entry of women into the labor market and promote greater involvement of men in caring for their children in most countries of Latin America and the Caribbean. So that both partners have a paid job and, in turn, exercise their shared parental responsibilities, it is required to extend to male workers care guarantees that are not exclusively linked to the biological reproductive role of women: pregnancy, childbirth and lactation. In that sense, paternity and parental leaves are useful tools to advance from the world of work towards overcoming the old model of "the male breadwinner and female housewife." However, these important changes alone are not enough. The implementation of positive actions is essential to stimulate the cultural change needed in order to foster a new perception of workers regarding family commitments

Keywords: parenthood, paternity, gender, work, care. 
MCS - Masculinities and Social Change Vol. 5 No. 3 October 2016

pp. 295-320

\section{Licencias de Paternidad y}

Permisos Parentales en América

Latina y el Caribe. Herramientas

Indispensables para Propiciar la

Mayor Participación de los

Padres en el Cuidado de los Hijos

e Hijas

Carina Lupica

Observatorio de la Matenidad

\section{Resumen}

En la mayoría de los países de América Latina y el Caribe se vuelve necesaria una reforma institucional que acompañe la incorporación de las mujeres al mercado de trabajo y promueva la mayor participación de los hombres en el cuidado de sus hijos e hijas. Para que ambos miembros de la pareja puedan trabajar de manera remunerada y, a su vez, ejercer las funciones parentales de manera compartida, se requiere extender a los trabajadores masculinos las garantías de cuidado que no están ligadas a la función exclusivamente reproductiva biológica de las mujeres: el embarazo, el parto y la lactancia. En ese sentido, las licencias de paternidad y los permisos parentales son herramientas útiles para avanzar, desde el mundo del trabajo, hacia la superación del viejo modelo "hombre proveedor y mujer dueña de casa". Sin embargo, estas importantes mutaciones no serán suficientes por sí solas. También se requiere la implementación de acciones positivas que posibiliten el cambio cultural necesario para fomentar una nueva percepción de los trabajadores ante los compromisos familiares.

Palabras clave: paternidad, maternidad, género, trabajo, cuidados.

2016 Hipatia Press

ISSN: 2014-3605

DOI: $10.17583 / \mathrm{MCS} .2016 .2083$ 


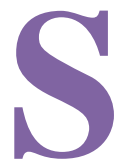

er padre es un proceso gradual que comienza con la decisión de tener y hacerse cargo de la protección, provisión y crianza de un niño o niña. Así, la paternidad incorpora la función proveedora material, la provisión de cuidados y la creación de lazos afectivos firmes y permanentes, que requieren mayor cercanía de los hombres con los niños/as (Oiberman, 1998; UNFPA-CEPAL, 2005).

Los hombres establecen con sus hijos/as una relación en el marco de una práctica compleja en la que intervienen factores sociales y culturales, que se modifican a lo largo del ciclo de vida del padre y de los niños/as. La paternidad es entonces un fenómeno cultural, social y subjetivo que relaciona a los hombres con sus hijos/as, más allá de cualquier tipo de arreglo conyugal (CEPAL, 2001, citado en Ugalde, 2008).

En diversas investigaciones se ha usado la expresión "trabajo del padre" en lugar de paternidad para centrarse en lo que los hombres hacen realmente en sus papeles paternales y también para enfatizar que el conjunto de habilidades y comportamientos que incluye el ejercicio de la paternidad, se pueden aprender (NCFR, 2003; Brown, 2004, citados en Lupica 2011).

La concepción y las características de las funciones paternas se han modificado a lo largo del tiempo, acordes con el desarrollo de las sociedades. En la actualidad, se asiste a un momento de mayor diversidad de estructuras y dinámicas familiares, dentro de las cuales conviven variados modos de concebir y ejercer la paternidad. Se puede ser un padre ausente, próximo o protagónico en la crianza de los hijos/as. Se puede ser padre por azar, sin haberlo elegido ni deseado (fundamentalmente, los adolescentes). Se puede ser padre no biológico ante la conformación de familias ensambladas, por reproducción asistida, en parejas del mismo sexo e incluso sin la presencia de la madre (Valdés, 2009).

Uno de los factores de mayor influencia en la historia reciente de la paternidad ha sido el ingreso masivo de las mujeres al mercado de trabajo y los consecuentes cambios en las funciones de los miembros de la familia. A partir de la segunda mitad del siglo XX, se produce un incremento de la incorporación de mujeres casadas y con hijos/as pequeños en la fuerza laboral, como causa del mayor nivel educativo de las mujeres y de la coyuntura económica en la región, que las impulsó al ámbito productivo 
para sostener económicamente sus hogares o paliar los ingresos masculinos que se habían perdido o deteriorado.

Paralelamente, se acrecienta la demanda sobre los hombres para que asuman mayores responsabilidades en las tareas del hogar y el cuidado de los hijos/as. En esta tendencia también influyó el desarrollo de evidencia sobre la trascendencia de la paternidad activa o comprometida en el destino de los hijos/as y en los sentimientos de bienestar y felicidad para los propios hombres (Barker \& Verani, 2008).

Pero, ¿hasta qué punto esta pretensión de mayor participación de los hombres en los cuidados de sus hijos/as ha tenido un correlato en los comportamientos al interior de la unidad familiar? ¿En qué medida el rol del padre está siendo redefinido para incluir el paternaje ejercido por padres presentes, afectuosos, contenedores y nutrientes?

En rigor, estas mutaciones no han sido lineales ni parejas al interior de la diversidad de los grupos sociales. Más bien, recorrieron trayectos en diversas direcciones y a distintas velocidades, pero, hasta el momento, han resultado insuficientes. Pese a que los hombres han respondido de manera creciente a las normas sociales cambiantes sobre la paternidad, su internalización no ha resultado ser un proceso sencillo. En la vida cotidiana, las actividades que conlleva el cuidado de los niños/as continúan estando muy fuertemente sesgadas por género, con una carga muy desigual que sigue siendo mucho más pesada del lado de las mujeres (Lupica, 2013).

\section{La Participación de los Hombres en el Cuidado de los Hijos/as ${ }^{1}$}

El trabajo que se realiza en los hogares, pero por el cual no se percibe ninguna remuneración -"trabajo doméstico no remunerado"- se ha denominado "trabajo reproductivo" debido a su aporte a la reproducción de la sociedad, ya que incluye el trabajo de cuidado. El cuidado incluye todo lo que hacemos para sostener la vida, para mantener, continuar y reparar nuestro nuestros cuerpos, nuestro ser y nuestro ambiente, de manera que podamos vivir tan bien como sea posible (Tronto, 2007, citado en Lupica, 2014).

El acto de cuidar, incluidas las labores domésticas que conlleva, se considera un trabajo porque implica tiempo, conocimientos, recursos y desgaste de energía y porque genera valor para quienes se benefician de él 
en forma directa y también para la sociedad (CEPAL, 2010, Ravazi \& Staab, 2010, citados en Lupica, 2014).

El trabajo de cuidado es realizado en distintos ámbitos y por personas diferentes. Puede ser desarrollado en la esfera doméstica, dentro de la propia familia, pero también incluye el trabajo que se ejerce en la esfera no doméstica, donde operan el Estado, las empresas, los organismos sin fines de lucro y las sociedades. Los roles y responsabilidades de los distintos actores intervinientes se superponen e interconectan en la compleja organización social del cuidado. El concepto de organización social del cuidado se refiere a la configuración que surge del cruce entre las instituciones que regulan y proveen servicios de cuidados a las personas y los modos como los hogares de distintos niveles socioeconómicos y sus miembros se benefician de ellos. Entender el cuidado como parte de la organización social supone prestar atención no solo a los aspectos microsociales sino también al rol de la política social en la provisión y regulación de las relaciones, actividades y responsabilidades de cuidado asignadas a las distintas instituciones y sujetos (Faur 2012, citada en Lupica, 2014).

En la historia y aún en la actualidad, la principal carga del trabajo no remunerado ha recaído en las familias, y en su interior, en las mujeres. Al asumir el trabajo no remunerado, ellas liberan a los trabajadores potenciales de la responsabilidad del cuidado. De esta manera, el trabajo no remunerado que ellas desarrollan influye sobre la cantidad de personas disponibles para el trabajo remunerado y sobre la cantidad de horas asignadas al mismo.

En todos los países de América Latina con información disponible, las mujeres en edad de trabajar dedican más del doble de tiempo que los hombres al trabajo doméstico no remunerado y, consecuentemente, pueden dedicar menos tiempo que ellos al trabajo remunerado. Pese a lo cual, su carga total de trabajo, que resulta de sumar el tiempo dedicado al trabajo remunerado y el no remunerado, es mucho mayor que el de los hombres (gráficos 1 y 2).

La sobrecarga de trabajo que sufren muchas mujeres genera altos costos para su desarrollo laboral, su salud y calidad de vida. Pero también repercuten negativamente en los cuidados que reciben los niños/as, en las relaciones de pareja, en la subutilización del potencial y aptitudes de las 
mujeres, lo que produce costos individuales (frustración), familiares (menores ingresos) y sociales (menores aportes a la productividad de las empresas y el país).

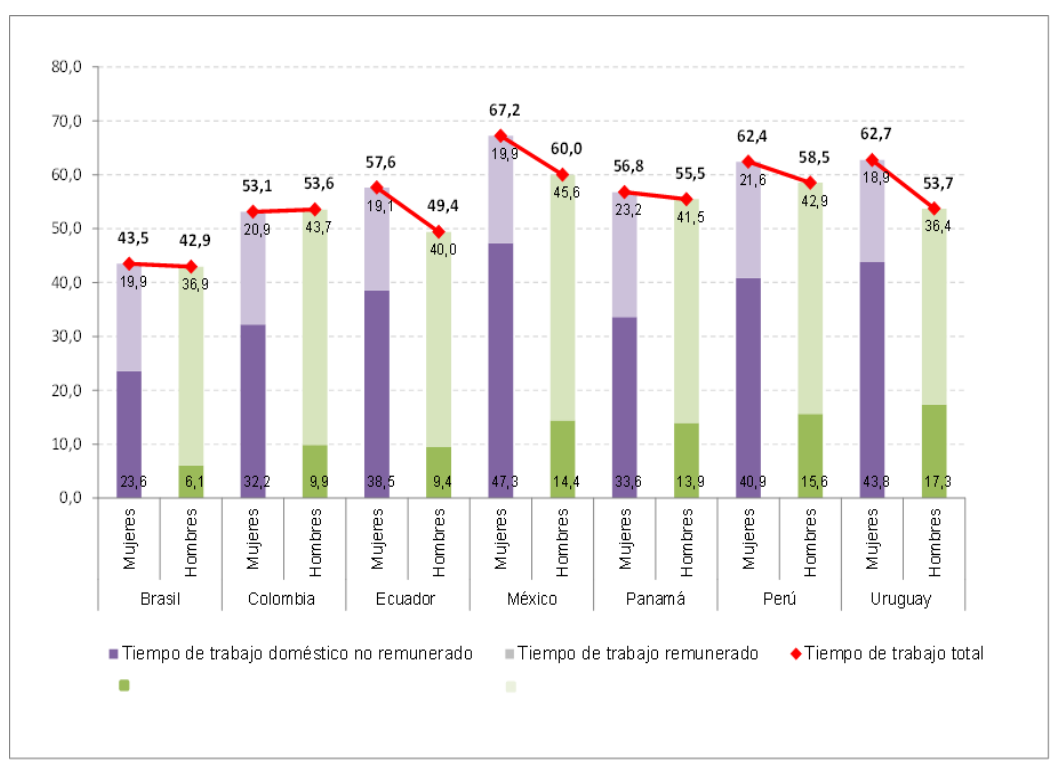

Gráfico 1. América Latina (7 países): tiempo destinado al trabajo total, remunerado y no remunerado. Población de 15 años de edad y más, por sexo, último año disponible (Tiempo en horas semanales).

Fuente: Comisión Económica para América Latina y el Caribe (CEPAL), División de Asuntos de Género, sobre la base de tabulaciones especiales de encuestas de uso de tiempo de los respectivos países. Brasil 2012, Colombia 2012, Ecuador 2012, México 2009, Panamá 2010, Perú 2010 y Uruguay 2007. 


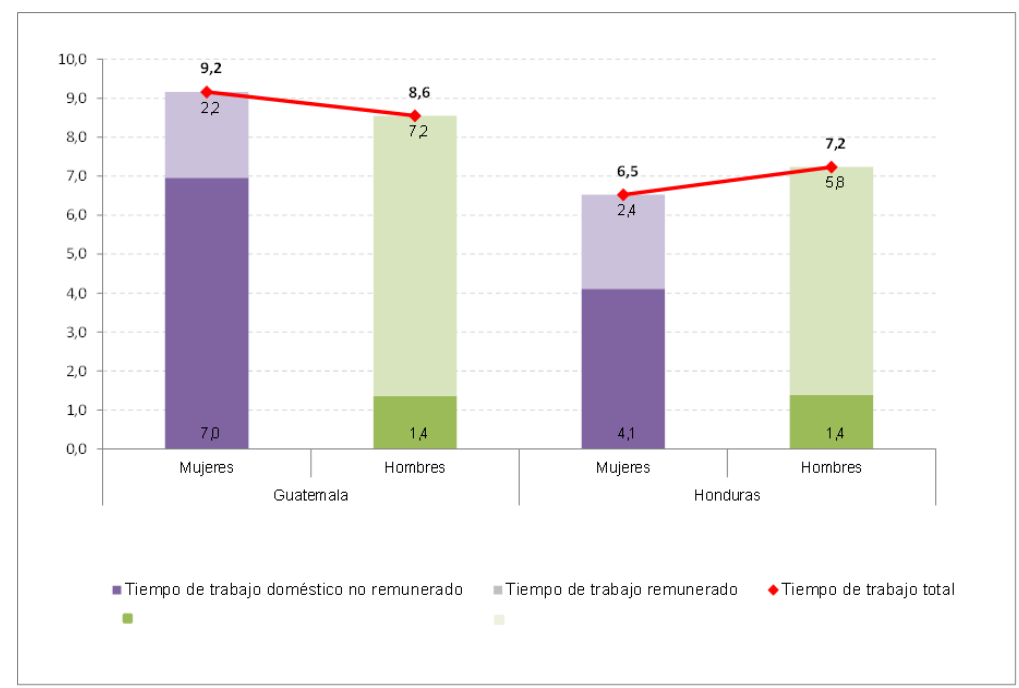

Gráfico $N^{\circ}$ 2: América Latina (2 países): tiempo destinado al trabajo total, remunerado y no remunerado. Población de 15 años de edad y más, por sexo, último año disponible (Tiempo en horas diarias).

Fuente: Comisión Económica para América Latina y el Caribe (CEPAL), División de Asuntos de Género, sobre la base de tabulaciones especiales de encuestas de uso de tiempo de los respectivos países. Guatemala 2011 y Honduras 2009.

La menor participación de los hombres en la crianza de los niños/as puede medirse también por su escasa presencia en las profesiones vinculadas al cuidado, tales como trabajadores del hogar, en centros de desarrollo infantil y escuelas primarias o cuidadores domiciliarios. En efecto, la atención profesionalizada o fuera del hogar de los niños/as es proporcionada mayoritariamente por mujeres. En Argentina, el 86\% de los trabajadores del cuidado son mujeres y una tercera parte de las mujeres trabajadoras lo hace en ocupaciones del cuidado, siendo la mitad de ellas maestras, profesoras, médicas y enfermeras, mientras la otra mitad son trabajadoras de casas particulares (Esquivel, 2010, citada en Lupica, 2014). 
Cuando los hombres participan en el cuidado de los hijos/as, lo hacen como un aporte o ayuda a las mujeres, no como una tarea de la cual se sientan responsables (Barker \& Verani, 2008). En Chile, la encuesta exploratoria "Padres del bicentenario" del año 2010 que realizó el Servicio Nacional de la Mujer entre 800 padres, indica que el $77 \%$ de los encuestados considera que cuando los hijos/as están en el hogar la madre es la principal responsable de su cuidado, el $11 \%$ destaca que son los abuelos y abuelas y, en tercer lugar, el $8 \%$ manifiesta que son ellos mismos. El 43\% de los hombres encuestados declara que no le dedica más tiempo al cuidado de sus hijos/as porque la madre lo hace y no le da espacio o nunca se lo ha pedido, el $11 \%$ declara no saber cómo cuidarlos, mientras que el $7 \%$ confiesa estar de acuerdo con la afirmación "son labores que no me corresponden" (SERNAM, 2012).

En suma, pese a que los hombres han flexibilizado sus roles sociales, el papel de cuidador no ha sido asumido en un plano de igualdad con las mujeres y, por ende, la reestructuración del uso del tiempo no se ha alcanzado exitosamente. El modelo patriarcal -hombre proveedor y mujer dueña de casa- ha demostrado ser muy resistente al cambio y, por consiguiente, los hombres no se han incorporado a las tareas de cuidado en el grado en que la nueva realidad social lo amerita (Hochschild, 2008, citado en Lupica, 2013).

\section{Redistribuir el Trabajo Reproductivo y los Cuidados entre Hombres y Mujeres}

La inequitativa distribución del trabajo reproductivo limita las posibilidades de las mujeres de participar en el mercado de trabajo en condiciones de igualdad con los hombres, contribuir con sus ingresos laborales al bienestar de sus familias y alcanzar su propia autonomía económica. A diferencia de ellos, las mujeres deben considerar las modalidades de concordancia entre el trabajo remunerado y no remunerado. En consecuencia, muchas elijen no participar en el mercado de trabajo o hacerlo en ocupaciones independientes, por cuenta propia o en jornadas parciales como una estrategia de conciliación, empleos que suelen ser de menor calidad, sin protección social y salarios inferiores (Lupica, 2015). 
Pero este no es el único motivo por el cual se deberían redistribuir estas tareas. La presencia del padre, dependiendo de su calidad, es positiva para los hijos/as y para los propios hombres. La paternidad presente y comprometida brinda mayores oportunidades para fortalecer el vínculo filial entre el padre y sus hijos/as, mejora el desarrollo de su estructura psíquica y emocional y les brinda la oportunidad de ser cuidados por ambos progenitores. Además, beneficia a los propios padres, para quienes la relación filial es una de las más importantes fuentes de bienestar y felicidad (Barker \& Verani, 2008).

Adicionalmente, en el ámbito social, la promoción de la parentalidad es un avance simbólico muy importante para la igualdad de género, pues constituye una forma de construir un modelo alternativo de maternidad y paternidad, de asignar un nuevo significado al papel de los padres dentro de los hogares como personas afectivas, interesadas y capaces de asumir responsabilidades familiares. Al mismo tiempo que contribuirá a transformar la concepción de maternidad "intensiva" y asociada al sacrificio y la omnipresencia frente a los hijos/as, juicio social que también dificulta la repartición de las tareas de cuidado y aliviar la sobrecarga que recae sobre las madres (Lupica, 2013).

Redistribuir el trabajo no remunerado entre los hombres y las mujeres de manera más equitativa es un objetivo que está incorporado en la Declaración y Plataforma de Acción de Beijing: “(...) la distribución equitativa entre hombres y mujeres de las responsabilidades respecto de la familia y una asociación armoniosa entre ellos son indispensables para su bienestar y el de su familia, así como para la consolidación de la democracia" (punto 15 de la Declaración de Beijing). También, la Plataforma de Acción establece entre sus objetivos estratégicos "Fomentar la armonización de las responsabilidades de las mujeres y los hombres en lo que respecta al trabajo y la familia" (objetivo estratégico F6). Además, este tema fue recogido por los distintos consensos de la Conferencia Regional sobre la Mujer de América Latina y el Caribe, en particular durante los consensos de Quito (2007) y Brasilia (2010) y el consenso de Montevideo de la Primera Reunión de la Conferencia Regional sobre Población y Desarrollo de América Latina y el Caribe (2013) (CEPAL, 2015).

Si bien estos acuerdos han permitido importantes mejoras en los países de la región, no han logrado superar la persistente brecha de género en la 
asunción del trabajo reproductivo, por lo que sigue siendo imprescindible promover cambios que apunten hacia una sociedad en la que las mujeres y los hombres sean a la vez cuidadores y trabajadores remunerados.

Entre las principales causas de las resistencias a la reestructuración de los tiempos dedicados por las mujeres y los hombres al trabajo para el mercado y en los hogares sobresalen dos principales: por una parte, existe una identificación histórica de los hombres con el trabajo remunerado y de las mujeres con el trabajo reproductivo. Por otra parte, la organización tradicional del trabajo para el mercado también dificulta el mayor compromiso de los hombres con el cuidado. Basta analizar el ordenamiento jurídico laboral de los países de la región para comprobar que fue creado para un trabajador masculino del sector industrial, a jornada completa, responsable del sustento económico familiar, y que no precisa medidas de conciliación porque no se lo considera responsable de las tareas domésticas y de cuidado (Lupica, 2013).

Las disposiciones para facilitar la ejecución del trabajo de cuidado en la región se refieren casi exclusivamente a los derechos de las mujeres asumiendo su doble función de trabajadoras y madres- y casi nunca a los hombres - cuya función como trabajadores parece partir del supuesto de que existe una mujer que cubrirá las necesidades de cuidado de sus hijos/as-. Esta presunción se encuentra implícita en la distribución de licencias para las madres y los padres, y en la regulación sobre los espacios de cuidado infantil en los lugares el trabajo que continúan asociadas exclusivamente a las trabajadoras.

\section{El Apoyo al Cuidado Compartido en la Legislación Laboral}

La legislación laboral sobre protección de la maternidad y apoyo a los trabajadores con responsabilidades familiares es una herramienta fundamental para promover la asunción compartida del cuidado entre las mujeres y los hombres, desde el mundo del trabajo. Dichas normativas tienen el objetivo de prevenir la discriminación y garantizar el derecho a trabajar remuneradamente sin tener que renunciar a proveer el cuidado de los miembros de la familia que así lo requieran y pone al Estado como garante de un nivel básico de protección de los trabajadores/as (Lupica, 2011). 
Los convenios de la Organización Internacional del Trabajo (OIT) y sus respectivas recomendaciones han tenido un notable impacto en el marco conceptual y en las legislaciones internas de los países de América Latina y el Caribe. Los mismos, han ido evolucionando desde la protección de las mujeres en su papel de madres hacia el establecimiento de iguales derechos y oportunidades para los trabajadores de ambos sexos. El reconocimiento de la participación de las madres y de los padres en las responsabilidades familiares, como la protección de las mujeres y de los hombres frente a una discriminación a causa de sus responsabilidades familiares, están incorporados en los Convenios $\mathrm{N}^{\circ} 3$ (1919); $\mathrm{N}^{\circ} 103$ (1952); $\mathrm{N}^{\circ} 183$ (2000) sobre protección de la maternidad y en el Convenio $\mathrm{N}^{\circ} 156$ (1981) sobre los trabajadores con responsabilidades familiares.

En términos de tendencias, se verifica un mayor desarrollo relativo de las normas de protección de la maternidad en el trabajo: entre principios de los noventa y la primera década del dos mil se observa un desplazamiento gradual hacia períodos más largos de licencia por maternidad en todas las regiones del mundo, mejoraron en general los niveles de su remuneración y las fuentes de financiamiento se modificaron de aquellas que dependían sólo del empleador a sistemas mixtos o a cargo de la Seguridad Social (OIT, 2010 citado en Salvador, 2013).

La región de América Latina y el Caribe no constituye una excepción. Como se puede observar en la tabla 1, todos los países prevén licencias de maternidad e incluso varios las extienden a las madres adoptivas. La mayoría de los países cumplen con el C183 que indica que las prestaciones relativas a la maternidad deben ser costeadas por el Estado y no por los empleadores, con el fin de impedir que se discrimine a las mujeres en el mercado de trabajo por el incremento del costo de su contratación para el empleador asociado a su condición real o potencial de madres. En el 61,8\% de los países analizados (21 países sobre un total de 34) las prestaciones pecuniarias durante la licencia de maternidad están a cargo exclusivo de la Seguridad Social, en el 29,4\% (10 países) el régimen de financiamiento es mixto (Seguridad Social y empleadores) y solo en 3 países el financiamiento corre por cuenta de los empleadores exclusivamente (tabla 1).

Todos los países de la región legislan sobre la protección contra el despido de las trabajadoras por razones relacionadas con la maternidad, 


\section{Lupica Carina-Licencias de paternidad y permisos parentales}

fuero que cubre la duración de la licencia de maternidad y, en la mayoría de los casos, también el embarazo y el tiempo de lactancia (ILO, 2014). Además, la mayoría de los países garantizan el derecho de la trabajadora a volver al mismo puesto o a uno equivalente tras su licencia de maternidad, y se prohíbe la exigencia de un examen de embarazo a una mujer que solicita un empleo (excepto para actividades de alto riesgo) (Lupica, 2013).

Sin desconocer estos aspectos positivos, las legislaciones de protección de la maternidad presentan en la región algunas limitaciones que afectan las posibilidades de las mujeres para desarrollarse plenamente en el ámbito laboral y familiar.

En primer lugar, la normativa rige solo en el caso de las asalariadas formales, lo que genera grandes exclusiones e inequidades con relación a otras personas que se desempeñan en el sector informal, en pequeñas empresas, o en el trabajo doméstico remunerado. En varios países se excluyen expresamente determinadas categorías de trabajadoras en el ámbito de la legislación de maternidad, tales como las trabajadoras domésticas; las familiares del empleador o las mujeres que trabajan en empresas familiares; las trabajadoras ocasionales o temporeras y las trabajadoras agrícolas (Lupica, 2013).

En segundo lugar, el marco normativo laboral tiende a centrarse en el momento del embarazo y la recuperación tras el parto, en un tiempo muy acotado, que no permite responder a las demandas conciliatorias cuando los hijos/as crecen. Como se refleja en la tabla 1, en el 76,5\% de los países analizados (26 países de un total de 34) la duración de la licencia de maternidad se encuentra por debajo del límite mínimo de 14 semanas establecido por el C183 de la OIT, y solo tres países (menos del 10\%) establecen la extensión de la licencia a 18 semanas o más, tal cual se estipula en la R191. Sin embargo, es frecuente que las legislaciones nacionales permitan o prescriban cambios en la duración de la licencia de maternidad si durante el embarazo o el parto tiene lugar algún acontecimiento no habitual o inesperado, tal cual aconseja la OIT en el C183 y la R191 (Lupica, 2013).

La duración de la licencia de maternidad es crucial para que la mujer se recupere del parto y pueda regrese en buenas condiciones de salud al trabajo. Cuando dicha licencia es demasiado breve, muchas madres no se sienten preparadas para retomar la vida laboral e incluso abandonan la 
fuerza de trabajo. En contraposición, unos períodos muy prolongados de licencia, también pueden afectar la participación o promoción de las mujeres en el empleo remunerado, con las consiguientes penalizaciones salariales (ILO, 2014).

Además de no cubrir las necesidades de cuidado de los hijos/as de más edad, los marcos legales en la región no incluyen el cuidado de otros miembros de la familia directa, como los adultos mayores, enfermos o las personas con capacidades diferentes. La legislación laboral actual solo prevé una licencia entre uno y tres días, según los países, para los trabajadores/as en caso de muerte de padres o suegros. Así, un desafío para la región consiste en diseñar normas -hoy inexistentes- que posibiliten a los trabajadores y trabajadoras asumir el cuidado de sus progenitores, algo que ocurre cada vez con mayor frecuencia dado el acelerado proceso de envejecimiento de la población (Valenzuela, 2010).

\section{Tabla 1}

Licencias de maternidad y de paternidad en América Latina y el Caribe.

\begin{tabular}{|c|c|c|c|c|c|c|}
\hline \multirow[b]{2}{*}{ País } & \multicolumn{3}{|c|}{ Licencia de Maternidad } & \multicolumn{3}{|c|}{ Licencia de Paternidad } \\
\hline & $\begin{array}{c}\text { Duración } \\
\text { (en } \\
\text { semanas) }\end{array}$ & $\begin{array}{c}\text { Prestación } \\
\text { durante la } \\
\text { licencia }\end{array}$ & $\begin{array}{c}\text { Fuente de la } \\
\text { prestación }\end{array}$ & $\begin{array}{l}\text { Duración } \\
\text { (en días) }\end{array}$ & $\begin{array}{c}\text { Prestación } \\
\text { durante la } \\
\text { licencia }\end{array}$ & $\begin{array}{l}\text { Fuente de } \\
\text { la } \\
\text { prestación }\end{array}$ \\
\hline Antigua y & 13 & $100 \%^{1}$ & $\operatorname{mixto}^{9}$ & & No existe & \\
\hline \multicolumn{7}{|l|}{ Barbuda } \\
\hline Argentina & 13 & $100 \%$ & seg soc & 2 & $100 \%$ & empleador \\
\hline Bahamas & 12 & $100 \%$ & $\operatorname{mixto}^{10}$ & 7 & No pago & - \\
\hline Barbados & 12 & $100 \%$ & seg soc & & \multirow{4}{*}{ No existe } & \\
\hline Belice & 14 & $100 \%$ & $\operatorname{seg} \operatorname{soc}^{*}$ & & & \\
\hline Bolivia, Estado & 13 & $95 \%{ }^{2}$ & seg soc & & & \\
\hline \multicolumn{6}{|l|}{ Plurinacional de } & \\
\hline Brasil & 17 & $100 \%$ & seg soc & $5^{19}$ & $100 \%$ & empleador \\
\hline Islas Vírgenes & 13 & $66,7 \%$ & seg soc & & $* *$ & \\
\hline \multicolumn{7}{|l|}{ Británicas } \\
\hline Chile & 18 & $100 \%^{3}$ & seg soc & 5 & $100 \%$ & seg soc \\
\hline Colombia & 14 & $100 \%$ & $\operatorname{seg} \operatorname{soc}^{*}$ & 8 & $100 \%$ & seg soc \\
\hline
\end{tabular}




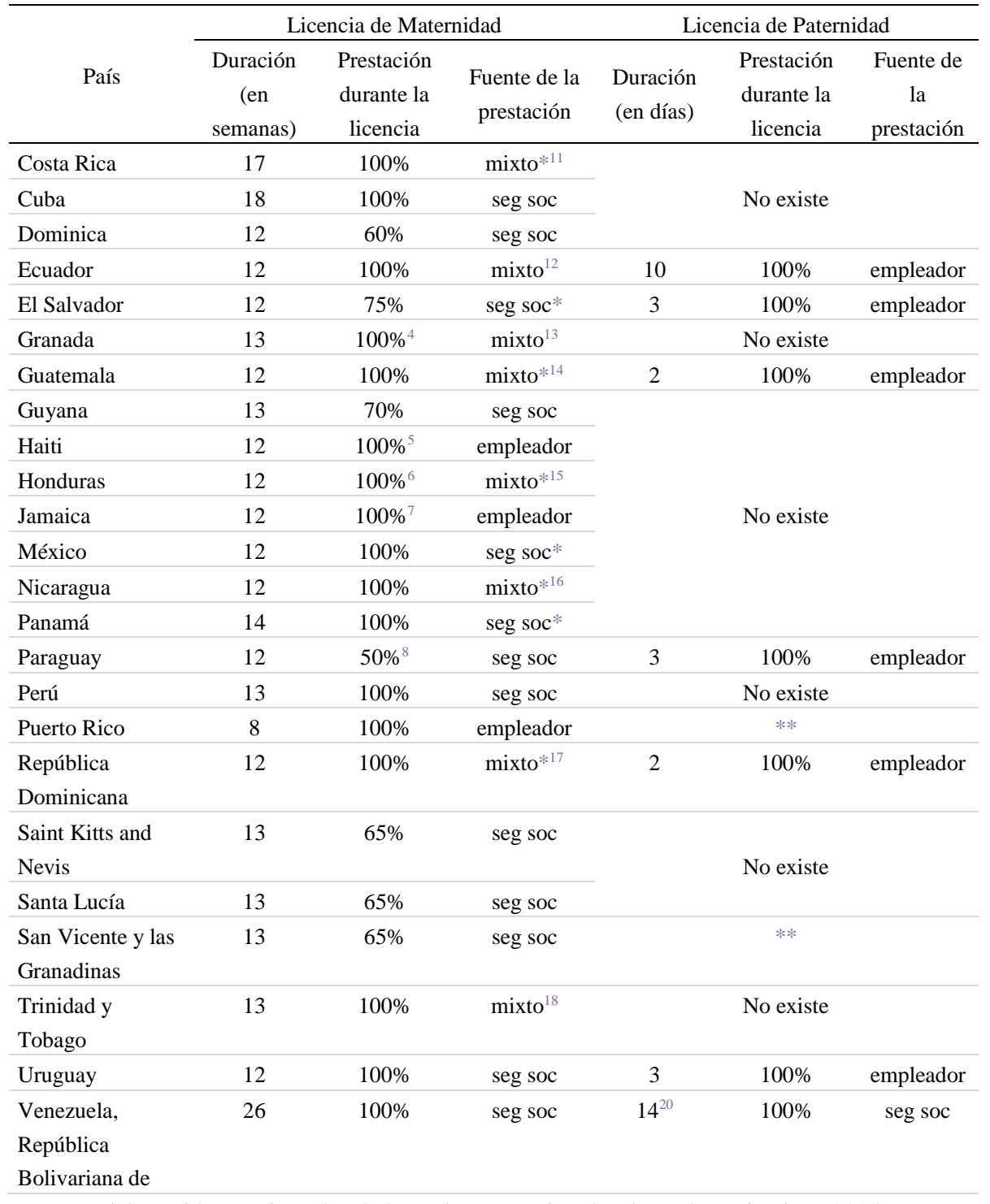

Fuente: Elaboración propia, sobre la base de International Labour Organization (2014).

Notas:

${ }^{1} 100 \%$ seis semanas y $60 \%$ siete semanas. 
${ }^{2} 100 \%$ para el salario mínimo y $70 \%$ para la diferencia entre el salario mínimo y el salario regular.

${ }^{3} 100 \%$ hasta un tope.

${ }^{4} 100 \%$ por dos meses y $65 \%$ para el último mes.

$5100 \%$ por seis semanas.

${ }^{6} 100 \%$ por diez semanas.

${ }^{7} 100 \%$ por ocho semanas.

${ }^{8} 50 \%$ por nueve semanas.

${ }^{9} 60 \%$ seg soc (seguridad social) y $40 \%$ el empleador por las primeras seis semanas.

$102 / 3$ seg soc por trece semanas y $1 / 3$ el empleador por doce semanas.

$1150 \%$ seg soc y $50 \%$ el empleador.

$1275 \%$ seg soc y $25 \%$ el empleador.

${ }^{13} 65 \%$ seg soc por tres meses y $35 \%$ el empleador por dos meses.

$142 / 3$ seg soc y $1 / 3$ el empleador.

$152 / 3$ seg soc y $1 / 3$ el empleador.

${ }^{16} 60 \%$ seg soc y $40 \%$ el empleador.

$1750 \%$ seg soc y $50 \%$ el empleador.

18 2/3 el empleador y $1 / 3$ seg soc.

195 días consecutivos.

${ }^{20} 14$ días consecutivos.

* Si la trabajadora no está cubierta por la seguridad social, pero califica para la licencia por maternidad, su empleador es responsable por el pago total del beneficio de la licencia de maternidad.

** Información no disponible.

Los marcos legales de apoyo al cuidado compartido presentan un menor nivel de desarrollo en comparación con la evolución de las normas de la protección de la maternidad en el trabajo. La legislación se ha desarrollado para las mujeres trabajadoras bajo el supuesto de que ellas son las principales responsables del cuidado familiar y no han integrado a los hombres, hecho que las perjudica frente a los empleadores que presumen que ellas representan un mayor costo laboral relativo. Tres ejemplos claros de esta inequidad:

i) Los hombres no tienen el derecho al "fuero paternal", lo que indica que pueden ser despedidos durante la gestación de su hijo/a incluso después del momento del parto, durante su licencia por paternidad. 
ii) La duración de la licencia por maternidad es de ocho a veintiséis semanas para las trabajadoras mientras que la licencia por paternidad es de apenas dos a catorce días. Además, la licencia por paternidad, cuando es remunerada, es principalmente financiada por el empleador y no por la Seguridad Social, lo que puede dificultar el ejercicio de ese derecho por parte de los hombres.

iii) En las reglamentaciones laborales de toda la región la oferta de servicios de cuidados en el lugar de trabajo está diseñado y restringido a las mujeres, debe ser provista por el empleador $\mathrm{y}$, en general, la obligatoriedad es proporcional al número de trabajadoras del establecimiento. De esta manera, se ignora la responsabilidad de cuidado del padre trabajador y se estimula a los empleadores a contratar menos mujeres que lo indicado por la norma. Por otra parte, la fiscalización del Estado muchas veces es insuficiente y es común el incumplimiento no sancionado de dicha obligación. Tampoco suele transferirse el equivalente monetario para que madres y padres trabajadores contraten servicios privados, donde la oferta es mayor, aunque estratificada de acuerdo a los ingresos de cada familia (Pautassi \& Rico, 2011).

Finalmente, las medidas laborales que incluyen el concepto de trabajador de ambos sexos con responsabilidades familiares son prácticamente inexistentes. Solo dos países en la región garantizan una licencia parental durante el período inmediatamente posterior a la licencia de maternidad: Cuba y Chile. En Cuba, los padres pueden decidir que uno de ellos quedará en casa cuidando a su hijo/a hasta que cumple un año de edad, a cambio de una retribución equivalente al $60 \%$ del beneficio de la licencia maternal. En Chile, a partir de la modificación del Sistema de Protección de la Maternidad en el año 2011, si ambos padres trabajan de manera remunerada, la madre puede decidir traspasar hasta seis meses de su permiso postnatal parental al padre, quien recibe un subsidio equivalente a su salario con un tope mensual. Este constituye el avance regional reciente más importante respecto a la normativa laboral a favor de la parentalidad (Lupica, 2015). 


\section{Licencias Parentales: Implementación y Resultados Recientes en Chile ${ }^{2}$}

El permiso parental o las licencias parentales consisten en un período de tiempo durante el cual un trabajador/a puede ausentarse de su puesto de trabajo con su reserva por el nacimiento, la adopción o tutela de un hijo/a para su cuidado, lo que garantiza la disponibilidad de tiempo y dinero para cuidar (Lupica, 2013).

Estas licencias forman parte de un destacado cambio jurídico caracterizado por la titularidad indiferenciada o neutra de los derechos de conciliación: se los reconoce como facultades individuales de los trabajadores, con independencia de su sexo. Ello conlleva la asunción de un modelo de familia con una doble fuente de ingresos provenientes del trabajo remunerado del hombre y de la mujer, más en consonancia con la realidad familiar actual (Caamaño, 2008).

Aunque las licencias parentales no figuran como tal en ninguno de los convenios de la OIT, el reconocimiento de la participación de los padres en las responsabilidades familiares en general y la licencia parental en particular están incorporadas implícitamente en la Recomendación $\mathrm{N}^{\circ} 191$ que acompaña al Convenio $\mathrm{N}^{\circ} 183$ y en la Recomendación $\mathrm{N}^{\circ} 165$ que acompaña al Convenio $\mathrm{N}^{\circ} 156$. En ambas recomendaciones se sostiene que tras la licencia de maternidad la madre o el padre deberían poder disfrutar de un permiso de licencia parental sin perder el empleo y conservando los derechos que se derivan de él (Lupica, 2013).

El Permiso Postnatal Parental (PPP) en Chile y su respectivo subsidio fueron establecidos por la Ley $\mathrm{N}^{\circ} 20.545$ en el año 2011 y forman parte del Sistema de Protección a la Maternidad contenido en el Código del Trabajo, en favor de todas las mujeres trabajadoras afiliadas al sistema previsional. Mediante la sanción de la ley, las trabajadoras tienen derecho a ausentarse del trabajo por un período total de doce semanas a continuación del período postnatal para cuidar del hijo/a recién nacido. Durante el tiempo en que se hace uso del permiso, la trabajadora recibe un subsidio por incapacidad laboral, que es un beneficio previsional (de la Seguridad Social) que consiste en una prestación de dinero que se paga en reemplazo de la remuneración de la trabajadora dependiente del sector público o privado, o en reemplazo de la renta de la trabajadora o trabajador independiente, siempre que cumplan los requisitos que exige la ley. 


\section{Lupica Carina-Licencias de paternidad y permisos parentales}

Las mujeres pueden elegir si tomar el postnatal a tiempo completo por una duración total de doce semanas o tomarlo a media jornada, en cuyo caso el tiempo se extiende a dieciocho semanas. Las mujeres que toman el postnatal a tiempo completo reciben un subsidio equivalente al $100 \%$ de su remuneración con un tope (a excepción de las funcionarias del sector público), mientras que la que lo toman a media jornada tienen derecho a un subsidio equivalente al $50 \%$ de su remuneración ${ }^{3}$.

Cuando ambos padres trabajan de manera remunerada, y a partir de la séptima semana del permiso, la madre puede traspasar parte de las semanas restantes al padre. Las semanas deben ubicarse al final del período de PPP y solo se pueden ceder semanas completas (siete días corridos), en la misma modalidad (a tiempo completo o media jornada) elegida por la madre. En consecuencia, si la madre decidió tomar doce semanas del PPP a jornada completa, puede traspasar al padre hasta un máximo de seis semanas a jornada completa, mientras que si ella decidió tomar dieciocho semanas a media jornada, puede traspasar al padre hasta un máximo de doce semanas, en media jornada. El PPP es independiente de la licencia por paternidad paga de cinco días para el padre al momento de nacer su hijo/a que sigue vigente.

Cuando el padre hace uso del PPP, el subsidio se calcula sobre la base de sus propias remuneraciones y también tiene un tope mensual, con excepción de los funcionarios públicos. El padre tendrá derecho a fuero laboral por el doble del período que se tome a jornada completa o a un máximo de tres meses si lo utiliza a jornada parcial, contados desde diez días antes de iniciarse el permiso.

De acuerdo a estudios previos a la implementación del PPP, los padres en Chile manifestaban tener deseos y voluntad de participar más en las tareas reproductivas. La encuesta Padres del Bicentenario del SERNAM, arroja que el $73 \%$ de los padres entrevistados consideraba necesario contar con más días de permiso cuando nace un hijo (SERNAM, 2011). Según los datos del Barómetro Mujer y Trabajo del año 2011, el 71,7\% de los hombres entrevistados, trabajadores dependientes de centros urbanos de la Región Metropolitana, manifestaron estar dispuestos a aceptar algunas semanas del PPP si la madre de su hijo/a estuviese dispuesta a transferírselo, el $18,7 \%$ declaró que no lo haría y el $9,6 \%$ no supo o no respondió (ComunidadMujer, Datavoz \& OIT, 2011). 
Pese a esto, una vez que el PPP comenzó a regir, fueron muy pocos los padres que hicieron uso del mismo. Desde noviembre de 2011 y hasta agosto de 2014 solo 713 madres traspasaron semanas del PPP al padre (el $0,3 \%$ del total de los subsidios iniciados), lo que representa un promedio de apenas 21 permisos mensuales.

Entre las razones que contribuyen a explicar el escaso uso del PPP por los padres, se pueden distinguir dos grandes grupos: las barreras culturales y sociales al cuidado compartido y algunos aspectos del diseño del permiso.

En relación a la primera dificultad, las expectativas culturales sobre la participación masculina en el cuidado de los hijos/as cumplen un rol muy importante. De acuerdo a los datos del Barómetro Mujer y Trabajo (2011), el 54,4\% de las mujeres entrevistadas antes de implementarse el PPP manifestaban disponibilidad a compartir parte del PPP con el padre de su hijo/a, el 40,3\% sostuvo que no lo haría y 5,3\% manifestó no saber o no respondió. Esto significa, que solo una de cada dos madres mostraba voluntad de compartir el cuidado de su hijo/a recién nacido con el padre.

En relación con las actitudes de los hombres, el resultado del estudio cuantitativo IMAGES (2011) revela que el 76,6\% de los hombres entrevistados antes de la implementación del PPP no se tomaron la licencia de paternidad (5 días), solo un 20,6\% señaló haber tomado una licencia pagada (derecho garantizado por normativa laboral a los trabajadores con contrato) y un 2,6\% declaró haber tomado una licencia no pagada. En cuanto a las principales razones que entregaron los hombres para no haber tomado el permiso postnatal, se observa que cerca de la mitad señaló que el trabajo no se lo permitió $(51,9 \%)$, mientras que un $13,9 \%$ manifestó que no tenía condiciones económicas para hacerlo y un 10,2\% señaló que simplemente no quiso tomarse el permiso (Aguayo, Correa \& Cristi, 2011).

A la luz de estos resultados, se constata la disonancia vigente entre los nuevos discursos y las viejas prácticas respecto a la división sexual del trabajo reproductivo, a la vez que era factible predecir que el uso del PPP por parte de los hombres no sería masivo.

A las visiones culturales tradicionales sobre quién debe encargarse de la atención y crianza los hijos/as pequeños, se suma la realidad del contexto socio laboral actual en Chile, caracterizado por extensas jornadas laborales, el miedo a perder el trabajo y el elevado nivel de endeudamiento de las 


\section{Lupica Carina-Licencias de paternidad y permisos parentales}

familias, lo que también representa un obstáculo importante para la mayor participación masculina en los cuidados.

En relación al diseño del PPP, éste fue concebido como una nueva licencia para las madres, quienes pueden cederla en parte y de manera voluntaria a los padres para el cuidado de sus hijos/as. Esto se contradice con la experiencia internacional, que indica que una de las características que contribuye a que la tasa de uso de los permisos parentales sea más igualitaria entre hombres y mujeres es el criterio de intransferibilidad o cuota parte de los permisos para hombres y mujeres, ya que los padres por lo general toman el tiempo de uso exclusivo dentro de la licencia parental (Lupica, 2013).

Adicionalmente, se produce una superposición del PPP con el tiempo recomendado de lactancia materna exclusiva durante los primeros seis meses de vida por la Organización Mundial de la Salud y el Fondo de las Naciones Unidas para la Infancia (UNICEF). De esta manera, los mensajes a las madres pueden resultar contradictorios: se recomienda la lactancia materna exclusiva durante los seis primeros meses de vida del niño/a y, por otra parte, se pretende que ella ceda parte de esos días al padre de su hijo/a.

Por otra parte, la tasa de uso de los permisos parentales está vinculada a la presencia o ausencia de otros instrumentos, recursos y servicios de cuidado y conciliación. Por tanto, es importante analizar las posibilidades y el funcionamiento integral de las licencias de maternidad, paternidad, parentalidad, por cuidado del hijo/a menor de un año grave y los servicios de cuidado como parte de un sistema de políticas de armonización favorables a las familias. En ese sentido también resulta paradójico promover el cuidado compartido desde el PPP pero excluir a los trabajadores masculinos en Chile de otros beneficios para el cuidado en el trabajo como la garantía de salas cuna, con excepción de los funcionarios públicos.

De esta forma, es loable concluir que con la creación del permiso postnatal parental en Chile se logró establecer un instrumento legal que posibilita el mayor involucramiento de los hombres en el cuidado de sus hijos/as, lo que constituye un progreso fundamental para el cambio cultural necesario a favor del cuidado compartido. A partir de ello, es recomendable fortalecer las acciones de difusión de la nueva ley y la promoción de la adopción del postnatal parental por parte de los padres. 


\section{MCS - Masculinities and Social Change, 5(3) 315}

\section{Reflexiones Finales}

Tal como se analizó a lo largo del artículo, con excepción de la reciente creación del permiso postnatal en Chile, el principal avance en las legislaciones laborales de los países de la región para promover los cuidados compartidos entre hombres y mujeres ha sido el establecimiento de la licencia de paternidad. La discriminación o restricción de los beneficios para los trabajadores masculinos reafirma a las mujeres en el rol de responsables casi únicas del cuidado del hogar y los niños/as, lo que conlleva un costo importante para las mujeres, los hombres y los niños/as.

Se vuelve necesario entonces introducir algunas modificaciones en los marcos normativos de la región para reflejar con mayor precisión y acompañar de manera más efectiva los cambios sociales y familiares acontecidos.

Entre las principales medidas, en cada uno de los países, se podrían analizar los costos y la factibilidad de extender la duración de las licencias por paternidad, incluir las licencias parentales remuneradas, legislar sobre licencias familiares para cuidar a otras personas dependientes que no sean los hijos/as pequeños y que puedan ser tomadas por las trabajadoras y los trabajadores e incorporar la posibilidad de excedencias para los hombres.

También, es importante modificar las normativas laborales para establecer la obligación de las empresas, indistintamente del sexo de sus empleados, de instalar, mantener o proporcionar centros de cuidado infantil en los lugares de trabajo. Los Estados deben fortalecer sus sistemas de fiscalización y sanciones por incumplimiento y estudiar las posibilidades de apoyar a las familias para la adquisición de servicios de cuidado en el sector privado, mediante subsidios o exenciones fiscales. Adicionalmente, desde las políticas públicas se debe promover la ampliación y regulación de los servicios de cuidado con el fin de aumentar su oferta, garantizar su calidad $\mathrm{y}$, al mismo tiempo, promover condiciones de trabajo decente para las y los trabajadores del cuidado.

Asimismo, el Estado debe profundizar la política de sensibilización y transformación cultural para incentivar la adopción de las licencias de paternidad y parentales por parte de los hombres, a través de distintas instancias, como campañas de información sobre la importancia y beneficios del ejercicio compartido de las funciones parentales para las 
familias, las empresas y la sociedad. El sistema educativo y los medios de comunicación son actores claves con los cuales se debería profundizar esta labor.

Finalmente, es necesario evaluar el uso efectivo de las licencias de paternidad por parte de los hombres e identificar los factores que facilitan o inhiben su práctica, con el fin de derivar lecciones y crear las condiciones para que se haga uso de ellas.

Las licencias de paternidad y los permisos parentales son herramientas útiles para modificar la inequitativa división sexual del trabajo. No obstante, las normativas por sí solas no bastan para alterar las pautas socioculturales predominantes. En ese sentido, se requiere de acciones positivas y de políticas públicas que posibiliten un cambio cultural para fomentar una nueva percepción del padre trabajador ante los compromisos familiares, tal como quedó demostrado con la experiencia reciente del permiso postnatal en Chile. Saldar esta deuda aún pendiente puede tener efectos positivos sobre la igualdad de género en el hogar y en el trabajo, y ser indicio de cambios en las relaciones y los roles de las madres y los padres, así como en los estereotipos de género predominantes en la región.

\section{Notas}

${ }^{1}$ Esta sección se elaboró sobre la base de Lupica (2014).

${ }^{2}$ Esta sección se elaboró sobre la base de Lupica (2015).

${ }^{3}$ A principios de 2016, en Chile se aprobó un proyecto de ley que perfecciona el permiso postnatal parental, ya que elimina el tope del subsidio y asegura el pago íntegro de la remuneración al funcionario o funcionaria del sector público durante la licencia. Asimismo, se contempla la habilitación del derecho de sala cuna en la entidad empleadora del padre cuando ambos sean funcionarios públicos. El proyecto estaba en etapa de promulgación al momento de finalizar la redacción de este artículo.

\section{Referencias}

Aguayo, F., Correa, P., \& Cristi, P. (2011). Encuesta IMAGES Chile. Resultados de la Encuesta Internacional de Masculinidades y Equidad de Género. Santiago de Chile: Cultura Salud/EME. Recuperado de: 
http://portales.mineduc.cl/usuarios/convivencia_escolar/doc/2012120 41625540.2011EncuestaIMAGESChileCulturaSaludEME.pdf Barker, G., \& Verani, F. (2008). La participación del hombre como padre en la región de Latinoamérica y el Caribe: una revisión de literatura crítica con consideraciones para políticas. Rio de Janeiro:

Promundo-Save the Children. Recuperado de: http://promundoglobal.org/wp-content/uploads/2015/01/Laparticipacion-del-hombre-como-padre-en-la-region-deLatinoamerica-y-el-Caribe.pdf

Biblioteca del Congreso Nacional de Chile-BCNC (2011). Historia de la Ley $N^{o}$ 20.545. Modifica las normas sobre protección a la maternidad e incorpora el permiso del Postnatal Parental. (D. Oficial de 17 de octubre, 2011). Recuperado de http://www.bcn.cl/catalogo/detalle_libro?bib=243276\&materia=Mate rnidad\%20--\%20Chile

Caamaño, E. (2008). El permiso parental y la progresiva inclusión del padre en los derechos para la armonización del trabajo y la vida familiar. Revista de Derecho de la Pontificia Universidad Católica de Valparaiso XXXI, 31, 325 - 354. doi:10.4067/S071868512008000200008

Comisión Económica para América Latina y el Caribe-CEPAL (2015). Informe regional sobre el examen y la evaluación de la Declaración y la Plataforma de Acción de Beijing y el documento final del vigesimotercer período extraordinario de sesiones de la Asamblea General (2000) en los países de América Latina y el Caribe. S. Montaño (Ed.). Recuperado de http://repositorio.cepal.org/bitstream/handle/11362/37718/S1421043 _es.pdf? sequence $=4$

ComunidadMujer, DataVoz, \& OIT (2011). III Barómetro Mujer y Trabajo. Recuperado de http://es.slideshare.net/comunidadmujer/iii-barmetromujer-y-trabajo-2011

Fondo de Población de las Naciones Unidas-UNFPA, \& Comisión Económica para América Latina y el Caribe-CEPAL (2005). Masculinidad y factores socioculturales asociados al comportamiento de los hombres: estudio en cuatro países de Centroamérica. M. Ortega, R. Centeno, \& M. Castillo (Eds.). 
318 Lupica Carina-Licencias de paternidad y permisos parentales

Recuperado de

http://www.berdingune.euskadi.eus/contenidos/informacion/material/ es_gizonduz/adjuntos/Masculinidad\%20y\%20factores\%20sociocultu rales \%20asociados\%20al\%20comportamiento\%20de $\% 20$ los $\% 20$ ho mbres\%20estudio\%20en\%20cua.PDF

International Labour Organization-ILO (2014). Maternity and paternity at work: law and practice across the world. Geneva. Recuperado de http://www.ilo.org/wcmsp5/groups/public/---dgreports/--dcomm/--publ/documents/publication/wcms_242615.pdf

Lupica C. (2015). Corresponsabilidad de los cuidados y autonomía económica de las mujeres: lecciones aprendidas del Permiso Postnatal Parental en Chile. Santiago de Chile: Naciones Unidas. Recuperado de:

http://repositorio.cepal.org/bitstream/handle/11362/37878/S1500262 _es.pdf?sequence $=4$

Lupica, C. (2014). Recibir y brindar cuidados en condiciones de equidad: desafios de la protección social en Argentina. Documento de trabajo $N^{\circ}$ 5. Buenos Aires: Organización Internacional del Trabajo-OIT. Recuperado de: http://www.ilo.org/wcmsp5/groups/public/--americas/---ro-lima/---ilobuenos_aires/documents/publication/wcms_302535.pdf

Lupica, C. (2013). Trabajo decente y cuidado compartido: hacia una propuesta de parentalidad. Santiago de Chile: Organización Internacional del Trabajo-OIT y Programa de las Naciones Unidas para el Desarrollo-PNUD. Recuperado de:

http://www.oitcinterfor.org/sites/default/files/file_publicacion/113B0 9_86_span.pdf

Lupica, C. (2011). Los padres que trabajan en la Argentina ¿tienen garantizado su derecho a cuidar? Paternidad y parentalidad en la legislación laboral. Boletín de la maternidad, 12, 15-19. Recuperado de http://www.o-

maternidad.org.ar/LinkClick.aspx?fileticket=WhFxczG0CrM\%3d\&ta bid $=132 \& \operatorname{mid}=753$

National Council on Family Relations (2003). Marriage Promotion in LowIncome Families. Retrieved from https://www.aafcs.org/res/NCFR_Marriage.pdf 
Oiberman, A. (1998). Padre-bebé. Inicio de una relación. La Plata:

Editorial de la Universidad de La Plata.

Olavarría, J. (2003). Los estudios sobre masculinidades en América Latina.

Un punto de vista. Anuario Social y Política de América Latina y el

Caribe, 6, 91-98. Recuperado de http://www.pasa.cl/wp-

content/uploads/2011/08/Los_Estudios_sobre_Masculinidades_en_A

merica_Latina_Olavarria_Jose.pdf

Pautassi, L., \& Rico, M. N. (2011). Licencias para el cuidado infantil.

Derecho de hijos, padres y madres. Desafíos. Boletín de la infancia y

adolescencia sobre el avance de los Objetivos de Desarrollo del

Milenio, 12, 4-9. Recuperado de

http://www.unicef.org/lac/desafios12_cepal-unicef.pdf

Programa de Naciones Unidas para el Desarrollo-PNUD (2010). Desarrollo

Humano en Chile 2010. Género: los desafíos de la igualdad.

Santiago de Chila: Programa de las Naciones Unidas para el

Desarrollo (PNUD). Recuperado de:

http://desarrollohumano.cl/idh/download/PNUD_LIBRO.pdf

Salvador, S. (2013). Análisis de costos y posibles impactos de diferentes

modelos de licencias por maternidad, paternidad y parentales.

Informe final de consultoría en el marco del proyecto URY2U503.

[online] Fondo de Población de las Naciones Unidas-UNFPA.

Recuperado de

http://www.unfpa.org.uy/userfiles/publications/76_file1.pdf

Servicio Nacional de la Mujer-SERNAM (2012). Estudio de los padres.

Participación en la crianza de los hijos. Recuperado de

http://estudios.sernam.cl/documentos/?eMjI2NDg1Ng==-

Estudio_de_los_Padres:_Participaci\%C3\%B3n_en_la_crianza_de_lo s_hijos_

Servicio Nacional de la Mujer-SERNAM (2011). Encuesta Nacional

Bicentenario 2011. Especial Familia, Maternidad y Crianza.

Recuperado de

http://estudios.sernam.cl/documentos/?eMjA2NzkxMg==-

Encuesta_Nacional_Bicentenario_2011_\%E2\%80\%93_Especial_Fa milia,_Maternidad_y_Crianza 
Ugalde, Y. (2008). Propuesta de indicadores de paternidad responsable. Naciones Unidas, Comisión Económica para América Latina y el Caribe-CEPAL.

Valdés, X. (2009). El lugar que habita el padre en Chile contemporáneo. Estudios de las representaciones sobre paternidad en distintos grupos sociales. Polis, Revista de la Universidad Bolivariana, 8(23), 385410. Santiago de Chile. Recuperado de http://www.scielo.cl/pdf/polis/v8n23/art17.pdf

Valenzuela, M. E. (2010). Trabajo y responsabilidades familiares en el contexto del envejecimiento: ¿Quién se encarga del cuidado? En A. Prado \& A. Sojo (Eds.), Envejecimiento en América Latina. Sistemas de pensiones y protección social integral (pp. 255-282). Santiago de Chile: Libros de la CEPAL 110.

Carina Lupica es miembro del Consejo de Asesores del Observatorio de Maternidad, Argentina.

Contact Address: Direct correspondence to Carina Lupica, Observatorio de Maternidad, Buenos Aires, email: lupica.carina@gmail.com 